\title{
Kala-azar Burden in Bangladesh- An Overview
}

\begin{abstract}
Sarkar $\mathrm{SR}^{1}$, Ray $\mathrm{NC}^{2}$, Kabir $\mathrm{MR}^{3}$
Abstract

Kala-azar is one of the major public health problems in Bangladesh and the disease is endemic for many decades. In SEA Region, VL is reported from 109 contiguous districts bordering Bangladesh, India and Nepal. Approx. 147 million people at risk in these three countries with an estimated 50,000 new cases each year. Kala-azar or Leishmaniasis is a disease caused by protozoan parasites of the Leishmania genus. It is transmitted by the sand fly named Phlebotomus argentipes and affects largely the socially marginalized and the poorest communities. The parasite migrates to the internal organs such as liver, spleen and bone marrow and if left untreated, will almost always result in the death of the host. Signs and symptoms include fever, weight loss, fatigue, anemia, and substantial swelling of the spleen and occasionally liver. In Bangladesh, kala-azar cases were reported from 45 of 64 districts, $>90 \%$ of cases were reported from just 10 districts. Mymensingh accounted for more than $50 \%$ of the total kala-azar cases reported in Bangladesh. Research in recent years has demonstrated the utility of non-invasive diagnostic modalities such as the direct agglutination test and rapid tests based on the immune response to the rK39 antigen. Primary kala-azar now can be easily recognized and effective treatment is available. Clinical trials in Bangladesh have reported encouraging results with amphotericin B (recommended as a third-line drug by the National Malaria Eradication Programme). Until a safe and effective vaccine is developed, a combination of sandfly control, detection and treatment of patients and prevention of drug resistance is the best approach for controlling kala-azar.
\end{abstract}

CBMJ 2020 January: vol. 09 no. 01 P: 43-50

Key words: Kala-azar, Leishmaniasis, Phlebotomus argentipes

\section{Introduction}

Visceral leishmaniasis (VL) or Kala-azar is considered as one of the most neglected diseases \& a re-emerging serious public health problem in the Indian sub-continent targeting the poor. ${ }^{+}$Nearly 350 million people are at risk in 88 countries around the world. Currently an estimated 12 million people are infected and around 2 million infections occur each year. ${ }^{2}$ Of all the cases, 90\% occur in India, Bangladesh, Nepal, Sudan and Brazil.

Leishmaniasis is caused by the protozoa Leishmania that are transmitted by sand flies of the genera Phlebotomus (Old World Leishmaniasis) and Lutzomyia (New World Leishmaniasis) obligate intracellular, kinetoplastoid protozoan parasites transmitted through the bite of infected sandfly. The clinical presentation ranges from simple cutaneous lesions to life threatening visceral forms. Visceral leishmaniasis is characterized by irregular bouts of fever, substantial weight loss, swelling of the spleen and liver, and anaemia (occasionally serious). If left untreated, the fatality rate in developing countries can be as high as $100 \%$ within 2 years. ${ }^{4}$

Kala-azar is significant health problem and has been reported from 45 out of 64 districts in Bangladesh. ${ }^{5}$ Kala-azar has resurged in endemic regions of Bangladesh since the 1990s, with the highest rates in the districts of Mymensingh, and Tangail. ${ }^{6}$ In Mymensingh specifically, the average annual incidence rate between 1994 and 2004 was $5.8 / 10,000$, and currently is as

1. Dr. Santana Rani Sarkar; Assistant Professor, Department of Microbiology, NMC

2. Dr. Nitai Chandra Ray; Assistant Professor, Department of Nephrology, $\mathrm{CBMCH}, \mathrm{B}$

3. Dr. Md. Rashedul Kabir; Department of Microbiology, CBMC, $B$

Address of correspondence:

E-mail: : nitairay@gmail.com Mobile: +8801716075694 
high as $300 / 10,000$ in the most affected communities. ${ }^{7,8}$

Bangladesh is committed to eliminate Kala azar by 2015 (Incidence of Kala-azar- less than 1 case in 10,000 populations at Upazila level). ${ }^{9}$ In May 2005, the three countries of South Asia Bangladesh, India and Nepal signed an MOU committing themselves to mutual cooperation towards elimination of kala azar from their respective countries. $^{4}$

A Regional Strategic Plan has been prepared and endorsed the guidelines and standard operating procedures (SOPs) to ensure the application of interventions in the endemic countries uniformly. The health facilities in KA affected areas are classified into different levels for programmatic purpose and by the diagnostic and treatment facilities available. Both the government and the private sector will be guided by the national guidelines, policy and norms. ${ }^{6}$

\section{History}

Kala-azar was first recognized as a specific disease in India. The term kala-azar was derived from Indian words Kala and Azar, meaning, "black sickness"; an illness in which the colour of the skin turns black. The word Kala also means "deadly"; thereby signifying fatal illness. ${ }^{10}$ The organism of VL or kala-azar was first described by Scottish docto Sir William Leishman. In 1903, Leishman identified certain organisms in smears taken from the spleen of a patient who had died from "dum-dum fever". At the time "Dum-dum", a town not far from Calcutta, was considered to be particularly unhealthy but in 1903 Irish physician Charles Donovan described them as being new. The link between these organisms and kala-azar was eventually discovered by Major Ross, who named them Leishmania donovani. In 1904, Leonard Rogers was first successful in culturing the parasite and showed that the flagellated form developed in culture. ${ }^{4}$ Today, the name kala-azar is used interchangeably with the scientific name visceral leishmaniasis for the most acute form of the disease caused by $L$. donovani.

\section{Prevalence}

Leishmania infections are worldwide in distribution: they are found in five continents. The disease is endemic in the tropical and temperate regions of 88 countries, 72 of them developing or least developed. ${ }^{11}$ Two million cases occur annually, however, there is a gross under reporting of the cases from endemic regions, and there has been a progressive increase in the case of leishmaniasis being reported from the newer areas. ${ }^{12}$ There are an estimated 500,000 new cases of $V L$ and more than 50,000 deaths from the diseases each year a death toll that is surpassed among the parasitic diseases only by malaria. ${ }^{13}$ Migration, lack of control measures and HIV-VL co-infection are the three main factors driving the increased incidence of VL. ${ }^{14,15}$ Since 1990, South Asia has experienced a resurgence of the lethal parasitic disease visceral leishmaniasis $(\mathrm{VL})$. The disease has been reported from 109 districts (45 in Bangladesh, 52 in India and 12 in Nepal) of three countries. ${ }^{16}$ The actual incidence rate of the disease is estimated to be about 8-10 times higher than the reported one in all three countries. ${ }^{17}$ Kala-azar appeared to have spread along the courses of the Ganges and Brahmaputra rivers. ${ }^{18}$

In Bangladesh, Mymensingh district reported 5 of 12 thanas kala-azar cases in recent years. Using the population of the respective Thana the denominator, the incidence of kala-azar in Fulbaria Thana ranged from 30 to 33/10,000/year since 2000, while that in Trishal, the next most affected Thana, ranged from 21 to $26 / 10,000 /$ year. Over the same period of time, the incidence in the other 3 endemic thanas, Bhaluka, Muktagacha, and Goforgaon, ranged from 5 to 15 cases/10,000/year. ${ }^{6}$

\section{Sources and Transmission}

Transmission of kala-azar is caused by the bite of infected female sandfly of the genera Phlebotomus and Lutzomyia. Some of the species feed on man and also a variety of 
warm and cold-blooded animals, which is an important factor in spreading the disease. A sandfly become infected 14-18 days after the ingestion of the infected blood meal and remains infected throughout its life time and are capable of infecting several persons. ${ }^{19}$ Transmission generally occurs in rural areas below $600 \mathrm{~m}$ from sea level, with a heavy annual rainfall, with a mean humidity above $70 \%$, a temperature range of $15-38^{\circ} \mathrm{C}$, abundant vegetation, subsoil water and alluvial soil. The disease is most common in agricultural villages where houses are frequently constructed with mud walls and earthen floors, and cattle and other livestock live close to humans. $^{20}$ Transmission of Kala-azar may take place by contamination of bite wound or contact when the insect is crushed during the time of biting. ${ }^{21}$

\section{Life cycle of Leishmania}

The sandfly injects the infective stage (ie, promastigotes) from its proboscis during blood meals. Promastigotes that reach the puncture wound are phagocytized by macrophages and other types of mononuclear phagocytic cells. In these cells progmastigotes transform into the tissue stage of the parasite (i.e., amastigotes), which multiply by simple division and proceed to infect other mononuclear phagocytic cells. Parasite, host, and other factors affect whether the infection becomes symptomatic and whether cutaneous or visceral leishmaniasis results. Sandflies become infected by in gesting infected cells during blood meals. ${ }^{22}$

\section{Pathogenesis}

The lifecycle of $L$. donovani has two distinct forms like a promastigote flagellar form found in the gut of the arthropod vector and an amastigote form, which develops intracellularly in the mammalian host. Only female phlebotomine sand flies transmit the disease, by inoculation of the promastigote form along with the sandfly saliva which potentiates parasite infectivity into the skin. The parasites are internalized by dendritic cells and macrophages in the dermis and transform into amastigotes by losing their flagella. They multiply and survive in phagolysosomes through a complex parasite-host interaction. ${ }^{23}$ The parasites disseminate through the lymphatic and vascular systems and infect other monocytes and macrophages in the reticulo-endothelial system, resulting in infiltration of the bone marrow, hepatosplenomegaly and sometimes lymphadenopathy. Importantly, infection does not always equate with clinical illness.

\section{Clinical Manifestations}

The three distinct forms, cutaneous leishmaniasis $(\mathrm{CL})$, visceral leishmaniasis $(\mathrm{VL})$, and mucocutaneous leishmaniasis (MCL) are classically caused by a spectrum of different Leishmania species each. Kalaazar (visceral leishmaniasis) is a disease that is insidious in origin, slow in development, and fearful in effects. Kalaazar is nearly always fatal if untreated. ${ }^{13}$ Even with treatment, case-fatality rates often exceed $10 \%$ in VL- endemic areas of Asia and Africa. ${ }^{24}$ The clinical features of VL caused by different species are different, and each parasite has a unique epidemiological pattern. ${ }^{25}$

Sub clinical infections \& PKDL patients are thought to exist at such a high number that they would be sufficient to maintain the infectious cycle as well as a reservoir. The incubation period varies from 3 to 8 months (range from 10 days to 34 months). Symptoms include fever, weight loss, hepatosplenomegaly (usually spleen much larger than liver), lymphadenopathy, pancytopenia and hypergammaglobulinaemia. ${ }^{26}$ Skin pigmentation may be a feature (kala-azar means black sickness). In advanced disease, death usually occurs because of severe secondary bacterial infections. $^{27}$ Post kala-azar dermal leishmaniasis (PKDL) develops after resolution of visceral leishmaniasis. In India, it manifests in 5-15 percent of VL cases after months or several years of remission from infection, while in Sudan, it develops within weeks or months in 50-60 percent of cured VL cases. The skin lesions are macular, maculo-papular or nodular, and usually spread from the 
perioral area to other areas of the body. ${ }^{26}$

\section{Complications}

Kala-azar is commonly complicated by secondary infections, such as pneumonia, bronchial infections, tuberculosis, malaria, diarrhoea or dysentery, viral infections, bacterial skin infections, otitis media and Cancarum oris. Thrombocytopenia may cause epistaxis or bleeding from other sites and this may precede death. Leishmania enteritis may be a cause of diarrhoea and malabsorption and pulmonary involvement may mimic pneumonia, Mortality is related to immunosuppression causing secondary infections and hemorrhage and in untreated cases mortality ranges from $75-95 \%$. ${ }^{28,29}$

\section{Host Defenses}

The host specific cell-mediated immune (CMI) response has an important role in controlling the infection. In VL patients, the inability to control $L$. donovani infection is associated with a profound T-cell unresponsiveness to $L$. donovani antigens and the production of interleukin 10. IL10producing CD25-Foxp3-T cells were recently implicated in the pathogenesis of human VL in India. ${ }^{30}$ The crucial role of the $\mathrm{CMI}$ response is illustrated by the increased risk of developing clinical illness in cases of malnutrition or concomitant immunosuppressive diseases, such as HIV infection. Other risk factors for developing clinical illness have been identified and include young age, decreased production of interferon $\mathrm{g}$ (IFN-g) and polymorphisms in the promoter of the tumour necrosis factor a (TNF-a) gene. ${ }^{31-33}$

\section{Laboratory diagnosis}

The diagnosis of $V L$ is complex because its clinical features are shared by a host of other commonly occurring diseases, such as malaria, typhoid, and tuberculosis; many of these diseases can be present along with VL (in cases of coinfection). Laboratory diagnosis of leishmaniasis can be made by the following: (i) Rapid dipstick test (rk-39 test); (ii) demonstration of parasite in tissues of relevance by light microscopic examination of the stained specimen, in vitro culture, or animal inoculation; (iii) detection of parasite DNA by PCR from blood or tissue samples; or (iv) immunodiagnosis (IFAT, ELISA, DAT) by detection of parasite antigen in tissue, blood, or urine samples, by detection of nonspecific or specific antileishmanial antibodies (immunoglobulin), or by assay for leishmania-specific cell-mediated immunity. ${ }^{11}$

\subsection{Specimen}

Specimens are Tissue sample (scraping, aspirate or punch biopsy), Bone marrow biopsy or splenic aspirate, Blood/Serum/Buffy coat, Urine.

\subsection{Immuno-chromatography test (ICT based rK39 dipstick test):}

A recombinant antigen, rK39 has been shown to be specific for antibodies in patients with Kala-azar caused by members of the $L$. donovani complex. ${ }^{34}{ }_{-36}$ Rk-39 antigen-based dipsticks using ICT method is rapid and cheap, it can be used for the reliable identification. The test has other potential values in detecting and typing parasites in vectors for epidemiological surveys.

\subsection{Microscopic examinations}

The commonly used method for diagnosing Kala-azar has been the demonstration of parasites in splenic or bone marrow aspirate. The presence of the parasite in lymph nodes, liver biopsy, or aspirate specimens or the buffy coat of peripheral blood can also be demonstrated. Amastigotes appear as round or oval bodies measuring 2 to $3 \mu \mathrm{m}$ in length and are found intracellularly in monocytes and macrophages. After identification, parasite density can be scored microscopically by means of a logarithmic scale ranging from 0 (no parasite per 1,000 oil immersion fields) to +6 (>100 parasites per field). ${ }^{37,38}$ 


\subsection{Culture of Leishmanial parasites}

Culture of parasite and improve the sensitivity of parasite isolation but Leishmania culture is rarely needed in routine clinical practice. However, cultures are required for (i) obtaining a sufficient number of organisms to use an antigen for immunologic diagnosis and speciation (ii) obtaining parasites to be used in inoculating susceptible experimental animals (iii) in vitro screening of drugs (iv) accurate diagnosis of the infection with the organism. Leishmania strains can be maintained as promastigotes in artificial culture medium. The culture media used may be monophonic (Schneider's insect medium, M199 or Grace's medium) or diphasic (NNN and Tobies medium). ${ }^{39}$ Usually prefer diphasic medium containing modified diphasic rabbit blood agar for primary isolation, and M199 medium containing $20 \%$ fetal calf serum to amplify parasite numbers.

\subsection{Specific serological tests}

\section{a) Antigen Detection}

The antigen detection is an ideal method of diagnosing an infection. Antigen levels are expected to broadly correlate with the parasite load as well. Antigen detection is more specific than antibody-based immunodiagnostic tests. ${ }^{40,41}$

\section{b) Enzyme Linked Immunosorbent Assay}

The Enzyme Linked Immunosorbent Assay (ELISA) is a valuable tool in the serodiagnosis of leishmaniasis. The test is useful for laboratory analysis as well as for field applications.

\section{c) Antibody detection}

Conventional methods for antibody detection included gel diffusion, complement fixation test, indirect haemagglutination test, IFA test, and countercurrent immuoelectrophoresis. ${ }^{42,43}$

\section{d) Indirect fluorescent antibody test (IFAT)}

The Indirect fluorescent test is one of the most sensitive tests available. The test is based on detecting antibodies which are demonstrated in the very early stages of infection and are undetectable six to nine months after cure. If the antibodies persist in low titers, it is a good indication of a probable relapse a probable relapse. Titers above 1:20 are significant and above 1: 128 are diagnostic.

\section{e) Direct agglutination test (DAT)}

The direct agglutination test is a highly specific and sensitive test. It is inexpensive and simple to perform making it ideal for both field and laboratory use. The method uses whole, stained promastigotes either as a suspension or in a freeze-dried form. ${ }^{45,46}$

\subsection{Molecular method}

Microscopy and culture have the limitations of low sensitivity and are time consuming. The immunological methods fail to distinguish between past and present infections and are not very reliable in immunocompromised patients. While the molecular approach is capable of detecting nucleic acids unique of the parasite, it would address these limitations. A variety of nucleic and detection methods targeting both DNA and RNA have been. The latest developments in molecular diagnostic technology have come about as a direct result of the advent of the polymerase chain reaction (PCR). The PCR is able to amplify small amounts of DNA or RNA to larger usable quantities developed. ${ }^{27}$

\section{Treatment of Kala-azar}

Agents with efficacy against visceral leishmaniasis (VL) include amphotericin B, pentavalent antimonial drugs, paromomycin (aparenteral aminoglycoside), and miltefosine (the first oral drug for treatment of VL). Conventional amphotericin B deoxycholate has high antileishmanial efficacy but is associated with high risk of renal toxicity and other side effects. Following drugs are recommended as 1st line treatment for KA in Bangladesh: 
The treatment of choice for visceral leishmaniasis acquired in Bangladesh is now Liposomal Amphotericin B (10 $\mathrm{mg} / \mathrm{kg} /$ single dose). Liposomal amphotericin $B$ is the drug with the highest therapeutic efficacy and the most favorable safety profile. AmBisome should be given in a single intravenous infusion at a dose of 10 $\mathrm{mg} / \mathrm{kg}$ for a period not less than 2 hours duration. Miltefosine, Paromomycin \& Combination treatment are alternative: Combination of Miltefosine and Paromomycin will be 1st choice. Other alternative combinations will be Liposomal Amphotericin B + Miltefosine or Liposomal Amphotericin B + Paromomycin LAmB 5 $\mathrm{mg} / \mathrm{kg}$ body weight on alternate days for 3 doses. Liposomal Amphotericin B is not indicated due to hypersensitivity, intolerance \& contraindication. At that time alternative drugs are use. ${ }^{5}$

\section{Prevention and control of Kala-azar infection}

Prevention and control of leishmaniasis require a combination of intervention strategies because transmission occurs in a complex biological system involving the human host, parasite, sandfly vector and in some causes an animal reservoir. Key strategies include:

12.1. Early diagnosis and effective case management reduces the prevalence of the disease and prevents disabilities and death. Currently there are highly effective and safe anti-leishmanial medicines particularly for $\mathrm{VL}$ and access to these medicines is improving. ${ }^{20}$

\subsection{Elimination of sandfly vectors:}

In endemic areas such as Bangladesh and India sandfly control is often combined with malaria control and Brazil with malaria and chagas disease control programmes. ${ }^{4,48}$ This is cost effective method given the expense of mass spraying with insecticides such as DDT, Malathion, Fenitrothion Diazinon and Dieldrin. In endemic areas this has to be an on-going process and continued surveillance is required to keep the vector populations low. 48

12.3. Control of reservoir hosts is complex and should be tailored to the local situation.

12.4. Education in the community mobilization and education of the community with effective behavioral change interventions with locally tailored communication strategies. Partnership and collaboration with various stakeholders and other vector-borne disease control programmes is critical at levels. ${ }^{20}$

\section{References:}

1. Joshi A, Narain JP, Prasittisuk C, Bhatia R, Hashim G, Jorge $A$ et al. Can visceral leishmaniasis be eliminated from Asia? Journal Vector Borne Dis 2008; 45: 105-11.

2. Rai ME, Muhammad Z, Sarwar J, Qureshi $A M$. Haematological findings in relation to clinical findings of visceral leishmaniasis hazara division. Journal Ayub Coll Abbottabad 2008; 3: 20-22.

3. Ahasan HN, Ayaz K, Bari SM. Current diagnosis and treatment of kala-azar Bangladesh perspective. Journal Medicine 2008; 9: 45-49.

4. WHO. 2009. Leishmaniasis: background information $A$ brief history of the disease [Online] Accessed on 2nd May, 2014 from http://www.who.int/leishmaniasis/history_dis ease/en/index.html

5. Bangladesh Ministry of Health and Family Welfare. National Guideline For Kala-Azar Cae Management, Kala-Azar Elimination Program, Dhaka: Communicable Disease Control Unit, DGHS, May, 2013.

6. Bangladesh Ministry of Health and Family Welfare. Kala Azar Elimination Programme, Mis: Technical Report Dhaka: Disease Control Unit, DGHS, 2010. 
7. Reithinger R, Dujardin J-C, Louzir H, Pirmez $C$, Alexander B, Brooker $S$. Cutaneous Leishmaniasis. Lancet 2007;7: 581-596.

8. Mandell Gl, Bennett Je, Dolin $R$, Eds. Mandell, Douglas, And Bennett's Principles and Practice of Infectious Diseases, $6^{\text {th }}$ Edition. Elsevier. 2005:2428-2442.

9. World Health Organization. Regional strategic framework for elimination of kalaazar from South East Asia region (2005-15) New Delhi, India: WHO SEAR; 2005.

10. Chatterjee KD. In: Parasitology (Protozoology and Helminthology), 12th edn, Chatterjee Medical Publishers, Calcutta. 1980; pp.53-69.

11. Sundar S, Rai M. Laboratory Diagnosis of Visceral Leishmaniasis. Clin. Diagn. Lab. Immunol. 2002; 9(5): 951-58.

12. Bora D. Epidemiology of visceral leishmaniasis in India. Natl Med J India 1999; 12: $62-68$.

13. Desjeux P. Leishmaniasis: current situation and new perspectives. Comp Immunol. Microbiol. Infect. Dis* 2004; 27: 305-18.

14. Boelaert M, El-Safi S, Mousa H, Githure J, Mbati $P$, Gurubacharya $V$ et al. Multicenter evaluation of repeatability and reproducibility of the direct agglutination test for visceral leishmaniasis. Trop. Med. Int. Health 1999; 4:31-37.

15. Desjeux $P$. The increase of risk factors for leishmaniasis worldwide. Transactions of the Royal Society of Tropical Medicine and Hygiene. 2001; 95 (3): 239-43.

16. Sundar S, Mondal D, Rijal S, Bhattacharya $S$, Ghalib $H$, et al. Implementation research to support the initiative on the elimination of kala azar from Bangladesh, India and NepalThe challenges for diagnosis and treatment. Trop Med Int Health. 2008; 13: 2-5.

17. Singh $S P$, Reddy DC, Rai $M$, Sundar $S$. Serious underreporting of visceral leishmaniasis through passive case reporting in Bihar, India. Trop Med Int Health. 2006; 11: 899-905.

18. Sanyal RK. Leishmaniasis in the Indian subcontinent. In: Chang KP, Bray RS, eds. Leishmaniasis. Amsterdam: Elsevier Science Publishers, B.V., 1985; p. 443-67.

19. Cheesbrough M. District Laboratory Practice in Tropical Countries, New York, USA, Combridge University Press. 2010.
20. WHO Leishmaniasis. 2014. Accessed on 15th May, .2014 from http://www. who.int/mediacentre/factsheets/f s375/en/.

21. Park JE, Park K. Park's Textbook of Preventive and Social Medicine. 16th ed. M/S Bannasidas Bhanot Publishers, Jabalpur, India. 2000; 231-33.

22. CDC Leishmaniasis. 2012; Available from http://www.cdc.gov/dpdx/leishmaniasis/inde x.html.

23. Contran RS, Kumar V, Robbins SL. Robbins Pathologic basis of disease, 5th edn, Prism books private limited, India1994.

24. Berman JD. Human Leishmaniasis: Clinical, Diagnostic and Chemotherapeutic Development in the last 10 years. Clinical Infectious Diseases 1997; 24: 684-703.

25. Siddig $M$, Ghalib $H$, Shillington $D C$, Petersen EA, Khidir S. Visceral Leishmaniasis in Sudan. Clinical features. Trop. Geogr. Med. 1990; 42: 107-112

26. Zijlstra EE, El-Hassan AM. Visceral leishmaniasis. Trans $R$ Soc Trop Med Hyg 2001; 95(Suppl 1): S27-58.

27. Wilson ME, Streit JA. Visceral leishmaniasis. Gastroenterol Clin North Am 1996; 25:535-51.

28. World Health Organization. Manual on Visceral leishmaniasis control. World Health Organization, Division of Control of Tropical Diseases, Geneva. 1996.

29. Peter CM. Leishmaniasis. In: Behrman RE, Kliegman RM, Jenson HB, editors, Nelson Textbook of Pediatrics. 16th edn. Philadelphia WB Saunders, 2000.

30. Nylen S, Maurya R, Eidsmo L, Manandhar $K D$, Sundar $S$, Sacks D. Splenic accumulation of IL-10 mRNA in T cells distinct from CD4+CD25+ (Foxp3) regulatory $T$ cells in human visceral leishmaniasis. J. Exp. Med. 2007; 204: 805817.

31. Zijlstra EE, el-Hassan AM, Ismael A, Ghalib HW. Endemic kala-azar in eastern Sudan: a longitudinal study on the incidence of clinical and subclinical infection and post-kala-azar dermal leishmaniasis. Am. J. Trop. Med. Hyg, 1994; 51: 826-836. 
32. Carvalho EM, Barral A, Sampaio DP. et al. Immunologic markers of clinical evolution in children recently infected with Leishmania donovani. J. Infect. Dis. 1992; 165: 535540 .

33. Karplus TM, SMB Jeronimo $S M B$, Chang $H$ et al. Association between the tumor necrosis factor locus and the clinical outcome of Leishmania chagasi infection. Infect Immun 2002; 70: 6919-6925.

34. Badaro R, Benson D, Eulalio MC, Freire $M$, Cunha S, Netto EM. rK39: a cloned antigen for Leishmania chagasi that predicts active visceral leishmaniasis. J. Inftect. Dis 1996; 173: 758-61.

35. Bern $C$, Jha $S N$, Joshi AB, Thakur GD, Bista $M B$. Use of the recombinant $k 39$ dipstick test and the direct agglutination test in a settingendemic for visceral leishmaniasis in Nepal. Am. J. Trop. Med. Hyg 2000; 63: 153-57.

36. Burns JM, Shreffler WG, Benson DR, Ghalib HW, Badaro R, Reed SG. Molecular characterization of a kinesing-related antigen of Leishmania chagasi that detects specific antibody in both African and American visceral leishmaniasis. Proc Natl Acad Sci USA 1993; 90: 775-90.

37. WHO. Control of Leishmaniasis. Report of a WHO Expert Committee. WHO Technical Report Series No. 793. WHO, Geneva. 1990; 1: 158.

38. Chulay JD, Brycesson ADM. Quantifications of amastigotes of leishmania donovani in smear of splenic aspirates of patients with visceral leishmaniasis. Am. J. Trop.Med. Hyg. 1983; 3: 2.

39. Sundar $S$, Pai K, Kumar $R$, Tripathi KP, Gam AA, Roy M. Resistance to treatment in kala-azar: speciation of isolates from Northeast India. Am J Trop Med Hyg 2001; 65: 193-96.

40. De Colmenares $M$, Portus $M$, Riera $C$, Gallego $M$, Aisa $M J$, Torras $S$ et al. Detection of $72-75 \mathrm{kD}$ and $123 \mathrm{kD}$ fractions of leishmania antigen in urine of patients with visceral leishmaniasis. Am. J. Trop. Med. Hyg. 1995; 52: 427-28.

41. Vinayak VK, Mahajan $D$, Sobit $R C$, Singh $N$, Sunder S. Anti-66 kDa anti leishmanial antibodies as specific immunodianostic probe for visceral leishmaniasis. Indian $J$ Med Res 1994; 99: 109-14.
42. Bray RS. Immnuodiagnosis of leishmaniasis. In: S. Cohen and E.H. Sadun (ed.), Immunology of parasitic infections, Blackwell Scientific Publications, Oxford, United Kingdom, 1985.

43. Haldar JP, Ghose S, Saha KC, Ghose AC. Serological profiles in Indian post kala-azar dermal leishmaniasis. Transaction of the Royal Society of Tropical Medicine and Hygiene 1981; 75: 514-17.

44. Davidson RN. Practical guide for the treatment of leishmaniasis. Drugs 1998; 56 : 1009-18.

45. Gari-Toussaint $M$, Leliever $A$, Marty $P$, LeFichoux $Y$. Contribution of serological tests of the diagnosis of visceral leishmaniasis in patients infected with the human immunodeficiency virus. Trans. $R$. Sco. Trop. Med. Hyg 1994; 88: 301-302.

46. Schallig HD, Schoone GJ, Kroon CC, Hailu $A$, Chappuis $F$, Veeken H. Development and application of simple diagnostic tools for visceral leishmaniasis. Med Microbiol Immunol (Berl) 2001; 190: 69-71.

47. World Health Organization. 13th Programme Report, Special Programme for Research and Training in Tropical Diseases, WHO Geneva, Switzerland. 1997; pp. 1-60.

48. Al-Masum MA, Evans DA, Minter DM, ElHarith A. Visceral leishmaniasis in Bangladesh: the value of DAT as a diagnostic too. Trans $R$ Sco Trop Med Hyg 1995; 89: 185-86. 